\title{
Herpes Simplex Virus Thymidine Kinase Gene Is Stably Maintained and Expressed in Cells Transformed by Protoplast Fusion
}

\author{
Rozanne M. Sandri-Goldin, ${ }^{1,3}$ Alan L. Goldin, ${ }^{1,4}$ Joseph Glorioso, ${ }^{2}$ and Myron Levine ${ }^{1}$ \\ 'Department of Human Genetics and ${ }^{2}$ Unit for Laboratory Animal Medicine, University of Michigan, Ann Arbor, \\ Michigan 48109
}

Received 25 August 1983-Final 28 November 1983

\begin{abstract}
We examined a series of transformed cell lines resulting from transfer of the herpes simplex virus type 1 thymidine kinase gene to Lt $k^{-}$cells by protoplast fusion gene transfer. We show that multiple copies of the transforming plasmid DNA, ranging from a minimum of two to greater than 20, were present in one or at most a few integration sites in each cell line. The $T K^{+}$phenotype was stable in five independent transformed cell lines after growth in nonselective medium for over a year. Transforming plasmid DNA was stable in one cell line containing from two to five copies after a year of growth in nonselective medium. In another cell line initially containing about 20 copies, the transforming DNA became rearranged soon after growth to mass culture, resulting in a decrease to two to five copies which then remained stably maintained. This suggests that $T K^{+}$transformants resulting from protoplast fusion are stable when the input DNA has integrated in a relatively low copy number.
\end{abstract}

\section{INTRODUCTION}

The transfer of purified genetic sequences to mammalian cells has proven to be a useful approach for the analysis of gene expression. A variety of techniques have been developed for the introduction of exogenous DNA into cells, and each technique has had different applications and results. The most widely used procedure is the DNA-calcium phosphate precipitation method of Graham and van der Eb (1), in which it is possible to introduce exogenous DNA into a large population of recipient cells (for review see reference 2). Direct microinjection (3-6), on the other hand, allows the introduction of a defined quantity of DNA into a more limited number of recipient cells. A third technique for gene transfer is protoplast fusion $(7,8)$, in which a large quantity of plasmid DNA can be quickly introduced into populations of recipient cells.

Since each of these techniques transfers DNA under different conditions, the uptake and integration of donor DNA may vary. In experiments in which DNA was transferred by the calcium phosphate precipitation method, large complexes of DNA which have been called pekelasomes or transgenomes were found in the recipient cell $(6,9-11)$. Perucho et al. (12) showed that carrier DNA was ligated to the transferred genes in these com-

\footnotetext{
${ }_{3}^{3}$ Present address: Department of Microbiology, University of California, Irvine, California 92717.

${ }^{4}$ Present address: Department of Chemistry, California Institute of Technology, Pasadena, California 91125.
} 
plexes. These concatenates can become integrated into one or a few sites in the host chromosome to form a stable transformant $(13,14)$ or may exist as independent units forming unstable transformants (10). Folger et al. (15) demonstrated that transformants isolated after direct microinjection could contain a single copy of the transforming gene or as many as 50-100 copies integrated in the cellular genome as a concatemer at one or a few sites, depending on the number of gene copies injected. Transformants isolated after protoplast fusion gene transfer have been shown to contain multiple copies of the transferred plasmid or cosmid sequences $(16,17)$.

In this report we extend the analysis of the integration patterns of transforming DNA transferred by fusion of animal cells with $E$. coli protoplasts. We found that multiple copies of plasmid molecules varying in number from 2 to greater than 20 were integrated into high-molecular-weight DNA. The transformed phenotype of the recipient cells was stable even after more than a year of passage in nonselective medium. We also report on the stability of the multiple copy number of integrated plasmid sequences from transformed cell lines maintained under different growth conditions for over a year of continual passage in culture.

\section{MATERIALS AND METHODS}

Isolation of $\mathrm{TK}^{+}$Transformed Cell Lines. Plasmid pX1 (18) cloned in E. coli strain DH-1 (19) was transferred to Ltk ${ }^{-}$cells (20) by protoplast fusion as described previously (7). Plasmid $\mathrm{pX} 1$ consists of the BamHI 3.5-kb fragment containing the HSV$1 t k$ gene inserted in pBR322. $\mathrm{TK}^{+}$colonies were selected after protoplast fusion by growth in medium containing HAT $[15 \mu \mathrm{g}$ hypoxanthine, $1 \mu \mathrm{g}$ aminopterin, $5 \mu \mathrm{g}$ thymidine, and $15 \mu \mathrm{g}$ glycine per milliliter (21)]. After 2 weeks, individual colonies were picked and grown to mass culture.

Analysis of stability of $T K^{+}$Phenotype. The stability of the $\mathrm{TK}^{+}$phenotype of the transformed cell lines was analyzed by following the protocol of Scangos et al. (10). $\mathrm{TK}^{+}$colonies were grown to $10^{6}$ cells in Eagle minimal essential medium supplemented with nonessential amino acids, $100 \mu \mathrm{g}$ streptomycin per milliliter, 100 units penicillin per milliliter, $10 \%$ fetal calf serum (Gibco), and HAT. At this time, termed day 0 , the cell lines were transferred to the same medium containing HT without aminopterin (nonselective medium). Cell lines were grown in HT medium for 10 days and were then transferred to medium without either HT or HAT. At day 0 and at various intervals thereafter (see Table 1), cells were plated at appropriate dilutions in HAT and HT media. Colonies were stained after 2 weeks and counted.

Transfer of Restriction EndonucleaseDigested DNA to Nitrocellulose and Hybridization. DNA isolated from each cell line after growth to mass culture or after a year of growth in HAT or unsupplemented medium was digested with the indicated restriction enzymes, electrophoresed in agarose slab gels, transferred to nitrocellulose, and hybridized to nick-translated probes as described previously (17).

\section{RESULTS}

Transformants Contain at Least One Complete Copy of Transferred Plasmid. Twelve $\mathrm{TK}^{+}$transformed cell lines were isolated by selection in HAT medium following protoplast fusion transfer to $\mathrm{Ltk}^{-}$cells of plasmid pX1 (18) which contains the HSV-1 $t k$ gene. To determine whether the HSV- $1 t k$ gene had integrated into the cellular genome in the $\mathrm{Ltk}^{+}$transformed cell lines, DNA was isolated from three of the cell lines, tk-1, tk-2, and tk-6, and analyzed by Southern transfer hybridization. Genomic DNA was digested with XbaI, which makes no cuts in the plasmid pX1; with HindIII which makes one cut; and with EcoRI which cuts the plasmid $\mathrm{pX} 1$ three times (Fig. 1). DNA from $\mathrm{Ltk}^{-}$cells and from plasmid $\mathrm{pX} 1$ mixed with $\mathrm{Ltk}^{-}$DNA was treated similarly. After transfer to nitrocellu- 


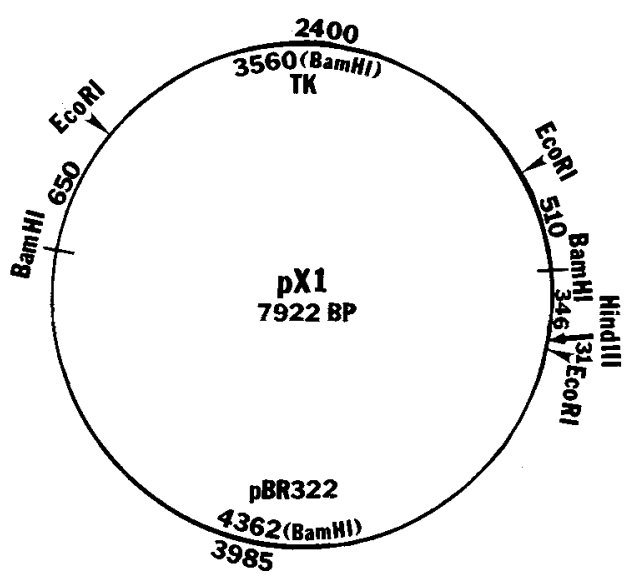

Fig. 1. Structure of plasmid pX 1 (18) which consists of a 3.5-kb BamHI fragment of HSV-1 containing the viral $t k$ gene inserted in pBR322.

lose, the filter was hybridized with the ${ }^{32} \mathrm{P}$ labeled 3.5-kb BamHI fragment of HSV-1 containing the $t k$ gene. Figure $2 \mathrm{~A}$ shows that a single high-molecular-weight band is present for each transformed cell line DNA cut with $\mathrm{XbaI}$. The bands from tk-1, tk-2, and tk-6 DNA migrate even more slowly than the $15.8-\mathrm{kb}$ supercoiled dimer form of plasmid $\mathrm{pX} 1$ seen in lane 2 . In our preparations of $\mathrm{pX} 1$ DNA, the plasmid was found predominantly in the dimer form. Since XbaI does not cut $\mathrm{pX} 1$, a band the size of $\mathrm{pX} 1$ DNA monomers $(7.9 \mathrm{~kb})$ or $\mathrm{pX} 1 \mathrm{DNA}$ dimers $(15.8 \mathrm{~kb})$ would be expected if the transferred plasmid DNA remained unaltered in these cells. The single bands of higher molecular weight observed in Fig. 2A suggest that the plasmid DNA had integrated into a single site in high-molecularweight DNA in these cell lines.

To determine whether all of the plasmid sequences were present in the cell lines, DNA was cut with HindIII or EcoRI and hybridized to the $3.5-\mathrm{kb} \mathrm{HSV}-1 \mathrm{tk}$ fragment. Figure 2B shows that, after HindIII digestion, a band of $7.9 \mathrm{~kb}$ is present in all three cell lines. This band is in the same position as the linear form of plasmid pX1 (lane 2). Additional bands probably representing the junction of plasmid and cellular DNA can also be seen. Figure $2 \mathrm{C}$ shows that all three bands generated by
EcoRI digestion of plasmid pX1 (Fig. 1) were present in each cell line, along with additional junction bands. These data indicate that at least one complete copy of the entire plasmid was present without noticeable rearrangements in each of these cell lines. This suggests that the plasmid DNA had integrated as a dimer or higher multimer, resulting in the presence of at least one complete, unrearranged copy of the plasmid sequences.

Based on the number of bands which likely represent the junctions of plasmid and cellular DNA, we can identify the probable site of integration within the plasmid molecules in each of these three transformed cell lines. Two bands of about $9.7 \mathrm{~kb}$ and $5.8 \mathrm{~kb}$ were seen in tk-1 DNA digested with HindIII (Fig. 2B). Because the hybridization probe consists only of HSV-1 tk sequences without pBR322 sequences, this indicates that both of these junction fragments contain HSV-1 sequences. This suggests that the integration event in the $\mathrm{pX} 1$ plasmid occurred within the HSV-1 sequences. Had the integration occurred in the pBR322 sequences, only one junction band would be expected. This is because the fragment from the HindIII site within pBR322 through the adjoining cellular DNA would not contain any HSV-1 sequences (Fig. 1) and hence would not hybridize with the HSV-1 tk probe. A similar conclusion can be drawn for cell line tk- 2 from the presence of two junction bands in the HindIII-digested DNA blot (bands of about $9.7 \mathrm{~kb}$ and $8.8 \mathrm{~kb}$ ) and also in the blot of EcoRI-digested DNA (a 4.3-kb and a $2.3-\mathrm{kb}$ band). In tk-6, only one possible junction band was present in both the HindIII and EcoRI digests, indicating that the integration event occurred within the pBR322 sequences in the plasmid. As discussed above, the second junction fragment consisting of pBR322 sequences along with cellular DNA was not detected with the HSV-1 probe. This second band was observed on rehybridization of the blot with a pBR322 probe (data not shown).

We also looked at the integration of plasmid $\mathrm{pX} 1$ in nine other transformed cell 
A

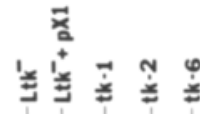

$15.8 \mathrm{~Kb}$

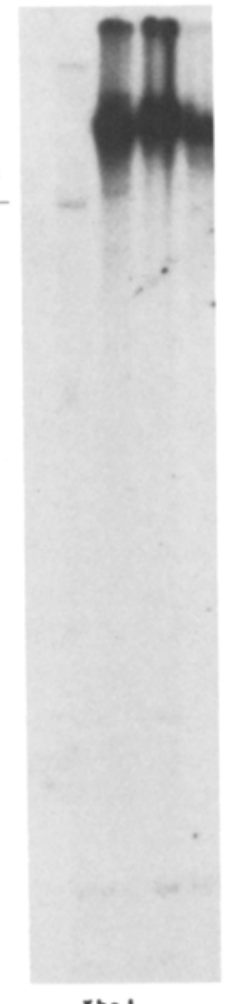

B
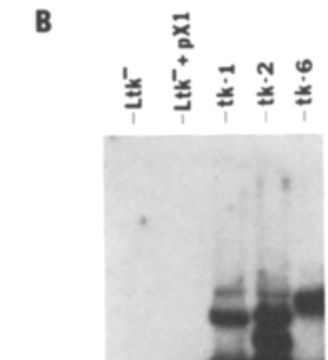

$7.9 \mathrm{~Kb}-$

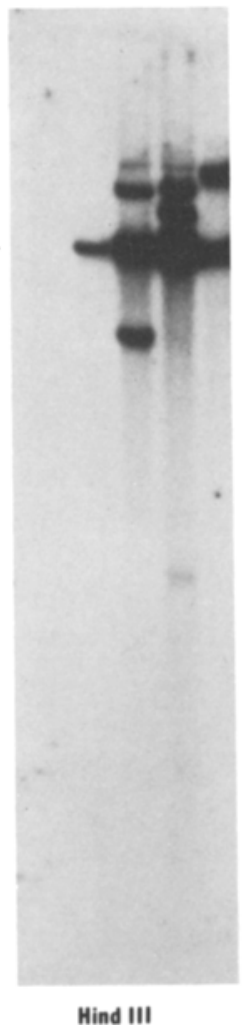

C
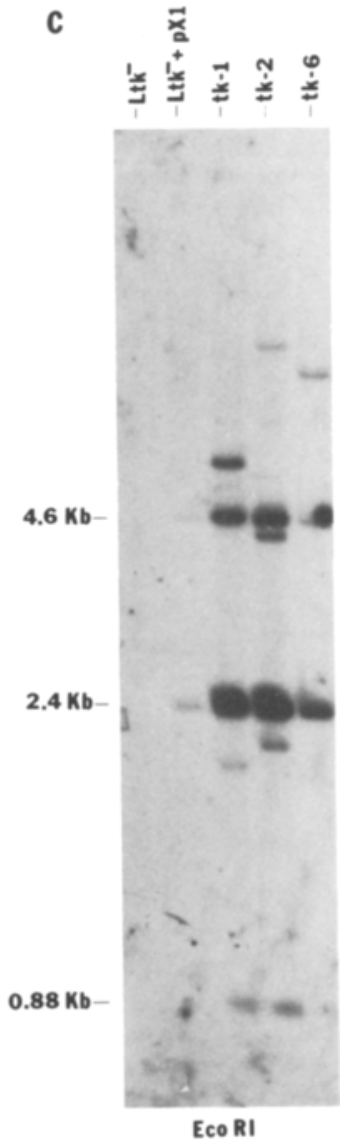

Fig. 2. Southern blot hybridizations of DNA from three $\mathrm{TK}^{+}$transformed cell lines. Twenty micrograms of (A) Xba I-digested, (B) HindIII-digested, and (C) EcoR I-digested DNA from cell lines tk-1, tk-2, and tk-6 were fractionated in $1 \%$ agarose and transferred to nitrocellulose (29). $\mathrm{Ltk}^{-}$DNA $(20 \mu \mathrm{g})$ and $20 \mathrm{pg}$ of plasmid pX1 DNA mixed with Ltk ${ }^{-}$ DNA $(20 \mu \mathrm{g})$ were treated similarly. Each blot was hybridized with the ${ }^{32}$ P-labeled 3.5-kb HSV-1 BamHI fragment of $\mathrm{pX} 1$. This fragment was purified by BamHI digestion of $\mathrm{pXI}$ followed by fractionation in low-melting-point agarose (Bethesda Research Laboratories). The 3.5-kb band was cut from the gel and the agarose was liquefied at $65^{\circ} \mathrm{C}$, then extracted with phenol four times and with ether four times. The DNA was precipitated with ethanol, resuspended in 10 $\mathrm{mM}$ Tris $\mathrm{HCl}, 1 \mathrm{mM}$ EDTA, $\mathrm{pH} 8.0$, and the purified fragment was labeled with ${ }^{32} \mathrm{P}$ by nick translation (30). The faint high-molecular-weight bands seen in digestions of tk-1 and tk-2 DNA with HindIII probably represent partial digestion products as they were not observed in subsequent experiments (see Fig. 3, lanes 2 and 3).

lines isolated by HAT selection after protoplast fusion transfer of $\mathrm{pX} 1$ to $\mathrm{Ltk}^{-}$cells. In blots of Xbal-digested DNA, a single highmolecular-weight band was seen for each transformant, suggesting a single integration into high-molecular-weight DNA (data not shown). When DNA from the transformed cell lines was digested with HindIII and hybridized with ${ }^{32}$ P-labeled pBR322 DNA, a band migrating with the 7.9-kb linear form of
pX1 was seen in each cell line (Fig. 3). Therefore, the entire plasmid was present in at least one intact copy in each cell line. The intensity of hybridization to the $7.9-\mathrm{kb}$ band differed in each cell line, suggesting that the plasmid sequences were present in different copy numbers in each transformant. One or two extra bands which could represent junction bands were also observed for these cell lines. 


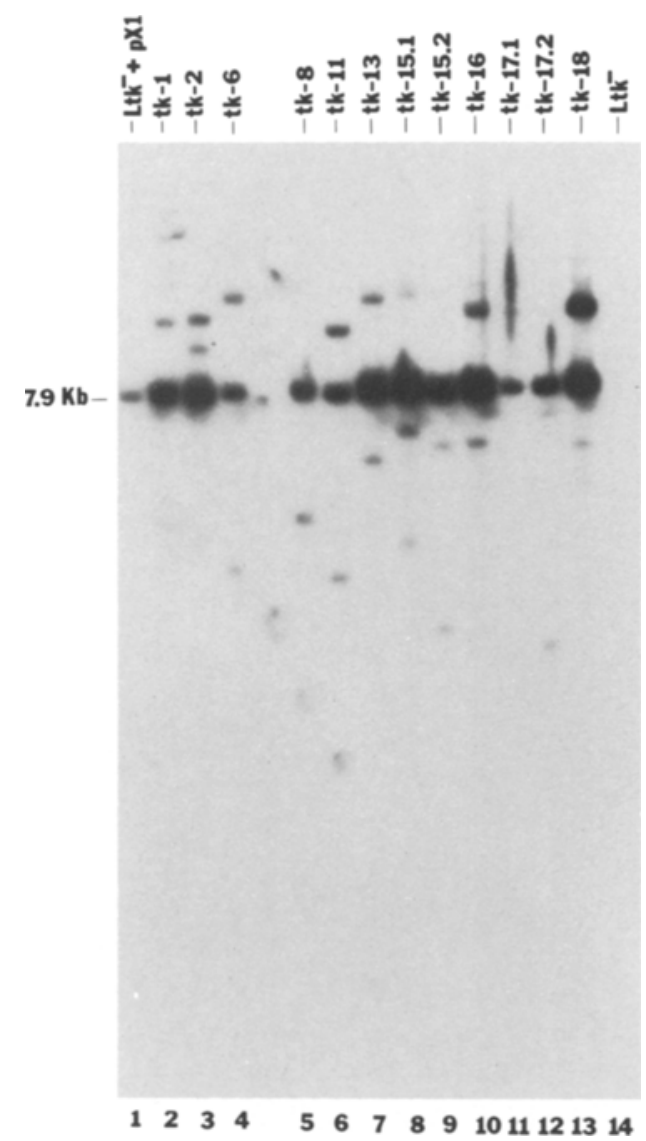

Fig. 3. Southern blot hybridizations of HindIII-digested DNA from $\mathrm{TK}^{+}$transformed cell lines. DNA $(20 \mu \mathrm{g})$ from $12 \mathrm{TK}^{+}$transformed cell lines (lanes 2-13) was digested with HindIII, fractionated in $1 \%$ agarose, and transferred to nitrocellulose. Ltk ${ }^{-}$DNA $(20 \mu \mathrm{g})$ (lane 14) and pXl DNA (20 pg) mixed with Ltk ${ }^{-}$DNA (lane 1) were treated similarly. The blot was hybridized with ${ }^{32}$ P-labeled pBR322 DNA.

Determination of Copy Number of $p X I$ Plasmid DNA in Ltk ${ }^{+}$Transformed Cell Lines. Since it appeared likely that plasmid pXl had integrated as at least a dimer in all of the transformants, we determined the copy number of $\mathrm{pX} 1$ sequences in cell lines tk-1, tk-2, and tk- 6 . A reconstruction experiment was performed in which $\mathrm{pX} 1$ DNA was mixed with Ltk ${ }^{-}$DNA in proportions equivalent to $1,2,5,7.5$, and 10 copies per haploid cellular genome and then digested with BamHI. Tk-1, tk-2, and tk-6 DNAs were also digested with BamHI. The blot was hybridized with gel- purified ${ }^{32} \mathrm{P}$-labeled HSV-1 tk fragment from $\mathrm{pX} 1$. The intact $3.5-\mathrm{kb}$ tk fragment of $\mathrm{pX} 1$ was seen in each cell line (Fig. 4). A comparison of the intensities of each band to the plasmid $\mathrm{pX} 1$ band in the reconstruction suggested that greater than 10 copies of the tk fragment were present in cell lines tk-1 and tk-2, whereas only about two copies of this fragment were detected in tk- 6 . Therefore, plasmid $\mathrm{pX} 1$ likely integrated as a dimer in the tk- 6 cell line and as a multimer of greater than 10 copies in cell lines tk-1 and tk-2.

Stability of $p X I$ Sequences in Transformed Cells. To determine whether the multiple copy number of plasmid sequences present in these transformed cell lines remains stable, we analyzed over time two cell lines, tk-8 and tk-15.1. The 7.9-kb HindIII fragment representing intact $\mathrm{pX} 1 \mathrm{DNA}$ is present in each cell line (Fig. 3, lanes 6 and 9). Genomic DNA shown in this blot was isolated as soon as the transformants wer grown to mass culture in HAT medium. Cell lines tk- 8 and tk-15.1 were then transferred to HT medium for 10 days. After that, half of each culture was placed in HAT medium and half in unsupplemented medium. The cultures in HAT medium were continually passaged for a year, representing over 100 passages under HAT selection. The cultures in unsupplemented medium were similarly passaged for a year under nonselective conditions. DNA was isolated from both the HAT selected and nonselected cultures at that time. This DNA, along with tk- 8 and tk-15.1 DNA from early passage cultures and $\mathrm{pX} 1$ DNA mixed with $\mathrm{Ltk}^{--}$DNA in proportions equivalent to $1,2.5$, $5,7.5,10$, and 20 copies per haploid cellular genome was then digested with BamHI, electrophoresed, and transferred to nitrocellulose. The blot was hybridized with ${ }^{32} \mathrm{P}$-labeled $\mathrm{pX} 1$ DNA (Fig. 5). Both the 3.5-kb tk fragment and the 4.4-kb nonselected pBR322 fragment were present in cell lines tk- 8 and tk-15.1 even after growth of the cultures under nonselective conditions for a year. A comparison of the intensities of the bands in the DNA from the three different tk-8 cultures shows that the 

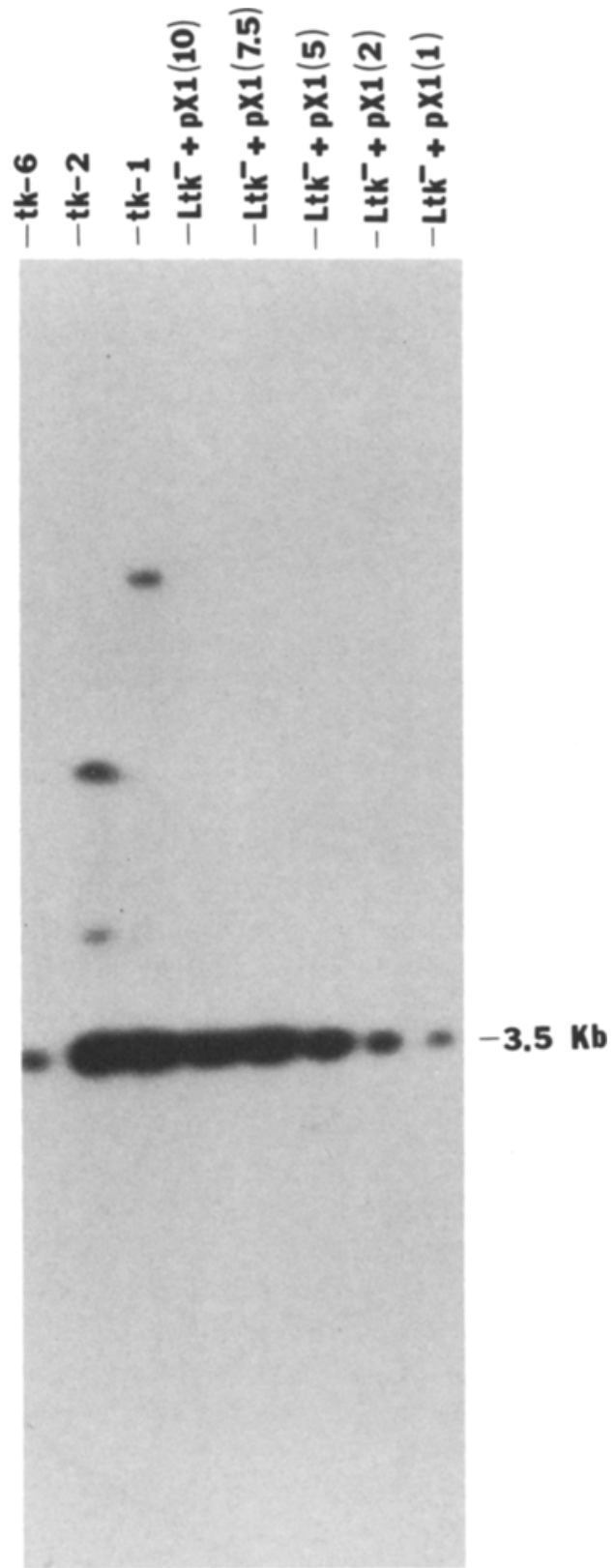

Fig. 4. Determination of the copy number of $t k$ sequences present in cell lines $t k-1, t k-2$, and tk-6. DNA $(10 \mu \mathrm{g})$ from each transformed cell line was digested with Bam$\mathrm{HI}$, fractionated in $1 \%$ agarose, and transferred to nitrocellulose. DNA from $\mathrm{pX} 1$ was mixed with $\mathbf{L t k}^{-}$DNA to approximate 1 copy per haploid cellular genome $(10 \mathrm{pg}$ pX1 per $10 \mu \mathrm{g} \mathrm{Ltk}^{-}$DNA), 2 copies/cell $(20 \mathrm{pg} / 10 \mu \mathrm{g}), 5$ copies/cell $(50 \mathrm{pg} / 10 \mu \mathrm{g}), 7.5$ copies/cell $(75 \mathrm{pg} / 10 \mu \mathrm{g})$, and 10 copies/cell $(100 \mathrm{pg} / 10 \mu \mathrm{g})$. The blot was hybridized with the ${ }^{32} \mathrm{P}$-labeled BamHI fragment of $\mathrm{pX} 1$ containing the HSV-1 $t k$ gene. This fragment was gel purified from $\mathrm{pX} 1$ as described in the legend to Fig. 2. copy number of the integrated plasmid sequences remained at about two to five copies per cellular genome. It is possible that an increase in copy number may have occurred in the culture grown in nonselective medium for a year as the bands from this sample appear to be more intense. In contrast, the copy number of $\mathrm{pX} 1$ DNA in cell line tk-15.1 decreased from about 20 copies per cell in the early passage sample to about two to five copies per cell in the cultures maintained under either selective or nonselective conditions for a year. Along with the decrease in copy number, the size of the two fragments representing the junction of plasmid and cellular sequences had also changed. Since DNA from both cultures showed the same banding patterns, this suggests that a rearrangement of the integrated plasmid sequences occurred early in these cells, after the early passage DNA was isolated but before the culture was divided into two cultures in either HAT medium or nonselective medium. After this rearrangement occurred, the integrated sequences remained stable, as did the integrated sequences in tk-8, for a year under the two different growth conditions.

Stability of th Expression in Transformed Cell Lines. Five cell lines were studied to determine whether the $t k$ genes present in the transformed cells continued to be expressed after growth of the culture in nonselective medium for various times. Cell lines tk-8, tk-15.1, tk-16, tk-17.1, and tk-18 were grown to mass culture after isolation in HAT medium and then transferred to HT nonselective medium for 10 days. The time of placement in HT medium was called day 0 . After 10 days, the cells were placed in medium containing neither HAT nor HT. At various intervals thereafter, dilutions of each culture were plated in HAT medium and in HT medium. Colonies were stained and counted two weeks after each plating. Table 1 shows that all five cell lines plated with nearly the same efficiency in both HAT and HT medium from day 0 up to over a year after transfer of the cell lines to nonselective medium. We 


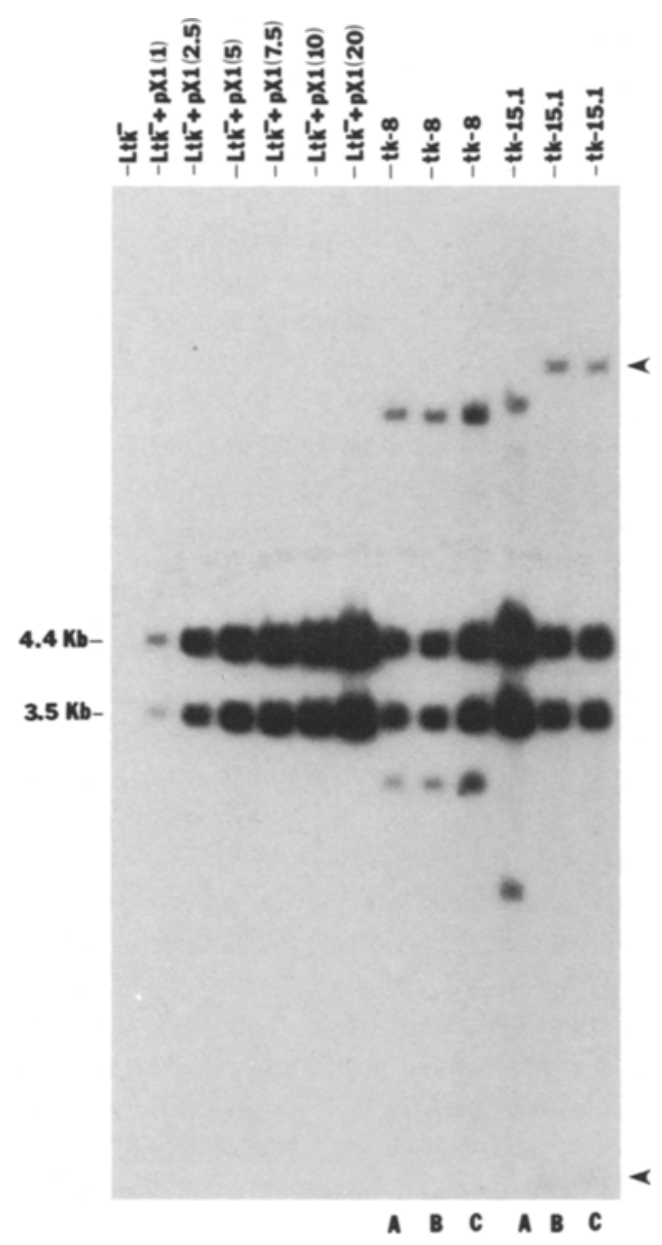

Fig. 5. Analysis of the stability of plasmid $\mathrm{pX} 1$ sequences in tk-8 and tk-15.1 transformed cell lines. DNA was isolated from tk- 8 and tk-15.1 cells as soon as they were grown to mass culture after isolation in HAT medium. Each cell line was then grown in HT medium for 10 days. At this time half of each culture was placed in HAT medium and half in unsupplemented medium, after which the cultures were passaged continually for a year. DNA was then isolated from each culture again. TK-8 and tk-15.1 DNA $(10 \mu \mathrm{g})$ was isolated early (A), after a year of growth in HAT medium (B), and after a year of growth in unsupplemented nonselective medium (C). The DNA was digested with BamHI, fractionated in $1 \%$ agarose, and transferred to nitrocellulose. To compare copy numbers of the plasmid sequences in these cell lines, $\mathrm{pX} 1$ DNA was mixed with Ltk $^{-}$DNA in proportions equivalent to $1 \mathrm{copy} / \mathrm{cell}(10 \mathrm{pg} / 10 \mu \mathrm{g}), 2.5$ copies/cell $(25$ $\mathrm{pg} / 10 \mu \mathrm{g}), 5$ copies/cell $(50 \mathrm{pg} / 10 \mu \mathrm{g}), 7.5$ copies $/$ cell $(75 \mathrm{pg} / 10 \mu \mathrm{g}), 10 \mathrm{copies} / \mathrm{cell}(100 \mathrm{pg} / 10 \mu \mathrm{g})$, and 20 copies/cell $(200 \mathrm{pg} / 10 \mu \mathrm{g})$ and similarly treated. The blot was hybridized with ${ }^{32}$ P-labeled pX1 DNA. The arrows show the positions of the junction bands found in tk-15.1 DNA isolated after a year of growth of cultures in HAT (B) or nonselective medium (C). Tk-8 DNA isolated after
Table 1. Stability of $t k$ Expression in Five Transformed $\mathrm{tk}^{+}$Cell Lines After Indicated Periods of Growth in Nonselective Medium ${ }^{a}$

\begin{tabular}{lllll}
\hline & & \multicolumn{3}{c}{$\begin{array}{c}\text { No. of days in nonselective } \\
\text { medium before plating } \\
\text { in Hat or HT }\end{array}$} \\
\cline { 3 - 5 } Cell line & Medium & 0 & 32 & 370 \\
\hline \multirow{2}{*}{ tk-8 } & HAT & $3.4 \times 10^{5}$ & $1.8 \times 10^{5}$ & $8.0 \times 10^{5}$ \\
& HT & $4.0 \times 10^{5}$ & $9.5 \times 10^{4}$ & $7.1 \times 10^{5}$ \\
tk-15.1 & HAT & $7.0 \times 10^{4}$ & $6.3 \times 10^{4}$ & $8.1 \times 10^{5}$ \\
& HT & $1.2 \times 10^{5}$ & $4.8 \times 10^{4}$ & $8.2 \times 10^{5}$ \\
tk-16 & HAT & $4.0 \times 10^{4}$ & $2.5 \times 10^{5}$ & $5.5 \times 10^{5}$ \\
& HT & $1.1 \times 10^{5}$ & $2.6 \times 10^{5}$ & $6.0 \times 10^{5}$ \\
tk-17.1 & HAT & $6.3 \times 10^{4}$ & $2.6 \times 10^{5}$ & $7.2 \times 10^{5}$ \\
& HT & $1.5 \times 10^{5}$ & $3.0 \times 10^{5}$ & $7.5 \times 10^{5}$ \\
tk-18 & HAT & $1.8 \times 10^{5}$ & $3.1 \times 10^{5}$ & $6.0 \times 10^{5}$ \\
& HT & $5.6 \times 10^{4}$ & $2.6 \times 10^{5}$ & $7.5 \times 10^{5}$ \\
\hline
\end{tabular}

${ }^{a}$ Cell lines were grown from colonies to $1 \times 10^{6}$ cells in medium containing HAT. At day 0 , cells were put into medium containing HT for 10 days after which the cells were grown in unsupplemented medium. At the times indicated, cells were plated at appropriate dilutions in HAT- or HT-containing medium. Two weeks after plating, colonies were stained and counted. The values shown were the total number of colonies counted for each time point multiplied by the dilution factor.

conclude that $t k$ expression was stable in these transformed cells.

\section{DISCUSSION}

In this report we show that protoplast fusion gene transfer of plasmid $\mathrm{pXI}$ to $\mathrm{Ltk}^{-}$ cells resulted in the integration of multiple copies of the entire plasmid into high-molecular-weight DNA. The plasmid sequences appeared not to be rearranged, with the multiple copies present in tandem head-to-tail arrangements. This conclusion is based on the absence of extra bands apart from the one or two expected junction bands observed for each cell line in the Southern hybridization experiments. Additional bands would have been expected, if the transferred plasmid sequences

a year of growth in nonselective medium (C) showed a slight increase in copy number of plasmid and flanking sequences. Since this increase was not observed in the sample from the culture maintained under selective conditions (B), it is likely that this apparent increase is due to the loading of slightly more cellular DNA in that slot. 
had joined together in a random array or if parts of plasmids were incorporated randomly in the multimers.

In studies of five transformed cell lines isolated after protoplast fusion transfer of a plasmid containing the $t k$ gene and the 16.5$\mathrm{kb}$ EcoRI $\mathrm{F}$ fragment of HSV-1, we also found integration of multiple plasmid copies into high-molecular-weight DNA (17). de Saint Vincent et al. (16) found integration of multiple copies of a cosmid containing a hamster $C A D$ (carbamyl-phosphate synthetase, aspartate transcarbamylase, and dihydroorotase) gene in cells transformed by protoplast fusion. They also demonstrated by in situ hybridization that the multiple-copy cosmids were integrated in the cellular chromosomes at one or at most a few sites. Folger et al. (15) found integration of multiple copies of a plasmid which had been microinjected into cells, and Huttner et al. (22) found integration of multimers consisting of full- or partiallength plasmid sequences in cells transformed by calcium phosphate transfection in the absence of carrier DNA.

While the mechanism for the formation of these multimers has not been elucidated, it is possible that the multimers were generated by homologous recombination of input plasmid molecules. Evidence demonstrating that homologous recombination between transferred plasmid molecules does take place has been presented recently $(15,23,24)$. While recombination between plasmids may have occurred after transfer, recombination was not observed between infecting HSV-1 genomes and homologous HSV-1 sequences integrated in mammalian cells $(17,25)$. This may indicate that the mechanism establishing multimers is preintegrative. Once exogenous sequences are integrated, they may be unavailable for homologous pairing, perhaps because of the chromatin structure they acquire.

Recombination events leading to large concatemers of input DNA may in fact facilitate integration of the exogenous sequences into the cellular genome. In calcium phos- phate transfections utilizing carrier DNA, large DNA complexes called pekelasomes or transgenomes were found in the recipient cell $(2,10,26)$. These complexes consist of transforming DNA ligated to carrier DNA. As mentioned previously, gene transfer without carrier DNA by calcium phosphate transfection, protoplast fusion, or microinjection all result in the generation of multimers of the input DNA. Folger et al. (15) did observe transformants containing a single copy of the transforming gene when the number of injected DNA molecules was low. They also noted that the frequency of transformation under these low copy number conditions was much higher if linear rather than circular molecules were injected. This suggests that free ends are important in the integration process. Homologous recombination leading to multimers of the input plasmid molecules may therefore promote integration by generating free ends during the breaking and rejoining of DNA molecules.

It should be noted that protoplast fusion not only transfers plasmid DNA molecules but probably also the bacterial chromosome into the recipient eukaryotic cells. It is possible that the bacterial DNA can act as carrier DNA. As stated earlier, we have not observed any extra bands in our Southern transfer experiments which could not be accounted for as plasmid sequences arranged in tandem multimers or as junction fragments. If bacterial DNA had become incorporated within plasmid sequences, new bands would be expected. As a test for the presence of $E$. coli DNA in the recipient cells we hybridized Southern blots identical to those shown in Figs. 2-5 with ${ }^{32} \mathrm{P}$-labeled E. coli $\mathrm{DH}-1$ DNA. No bands were observed in any of these hybridizations (data not shown). While this does not rigorously exclude the possibility of incorporation of some bacterial sequences because the complex DH-1 sequences are an insensitive hybridization probe, we can conclude that large amounts of bacterial DNA do not remain in the transformed cells.

Another explanation for the presence of 
multiple copies of the input plasmid DNA in the transformed cells could be gene amplification following integration. Reyes et al. (27) presented evidence that the HSV-1 $t k$ gene had integrated in Ltk ${ }^{-}$DNA early after transformation by calcium phosphate transfection, and that subsequently, both the viral $t k$ and the flanking cellular sequences were amplified to create up to 20 tandem duplications. Roberts and Axel (28) also observed gene amplification following $t k$ transformation, but these investigators selected for amplification by requiring reversion of the $\mathrm{tk}^{-}$phenotype to the $t k^{+}$phenotype.

We think it unlikely that gene amplification after integration was responsible for the multiple copy plasmid sequences integrated in our cell lines, because the junction fragments which should contain flanking sequences appeared to be present at the level of one copy per genome (Fig. 4 and 5), whereas the plasmid bands were present in multiple copies. If gene amplification had occurred, the copy number of both the plasmid and flanking sequences (as represented by the junction bands) would have increased. One exception which might be a case of amplification was cell line tk-18 (Fig. 3, lane 13). The tk-18 DNA showed a band of higher molecular weight than the plasmid band which could represent a junction band. This band was of nearly the same intensity as the plasmid band. It is therefore possible that gene amplification of plasmid and flanking sequences could have occurred in this cell line. Our data do not exclude the possibility that amplification could have occurred by homologous reciprocal exchanges involving only the amplified unit and not the flanking sequences. If this were the case, we could not distinguish between amplification by reciprocal exchanges within the repeat unit and an initial integration of a multimeric concatemer, as the final products would be the same.

Plasmid DNA sequences that are present in a high copy number may be be unstable in the transformed cell lines. Cell line tk- 8 contained around two to five copies of the plasmid
DNA in early passage cells, and the cells retained this copy number even after a year of growth. In contrast, cell line tk-15.1, which had about 20 plasmid copies at early passage, underwent a rearrangement resulting in a loss of plasmid copy number and formation of different junction bands. This rearrangement occurred shortly after the early passage DNA was isolated, after which the cell line contained between two and five copies of the plasmid. This copy number was stably maintained even after continual passage of the culture for a year under either selective or nonselective conditions. We have observed a similar decrease in plasmid copy number from about 20 to about two to five in two transformed cell lines containing a plasmid with the $t k$ gene and the EcoRI $F$ fragment of HSV-1 (Sandri-Goldin et al., manuscript in preparation). Reyes et al. (27) also observed that $t k$-transforming sequences decreased from about 20 to four copies per cellular genome in two transformed cell lines. A high number of copies may be unstable, perhaps because genetic material is lost by recombination between tandemly arranged sequences. However, the hamster $C A D$ gene transferred to recipient cells by protoplast fusion was found to be integrated at high copy number (50-100 per cell) with the sequences in a tandem array (16). The high copy number remained stable in those transformed cell lines. Therefore, it is likely that some other explanation accounts for the reduction in the $t k$ copy number. The possibility is that a high number of copies of the $t k$ gene may be unstable because high levels of thymidine kinase are deleterious to cells. If this were the case, cells which have undergone rearrangements resulting in lower copy number would have lower levels of thymidine kinase and would be selected.

\section{ACKNOWLEDGMENTS}

This work was supported by grants AI17900, AI18228, and RR02000 from the National Institutes of Health. A.L.G. was 
supported by Medical Scientist Training Program grant GM07863 and National Institutes of Health predoctoral training grant T32GM07544.

\section{LITERATURE CITED}

1. Graham, F.L., and van der Eb, A.J. (1973). Virology 52:456-467.

2. Scangos, G., and Ruddle, F.H. (1981). Gene 14:110.

3. Capecchi, M.R. (1980). Cell 22:479-488.

4. Diacumakos, E.G. (1973). Methods Cell Biol. 7:287-311.

5. Graessman, A., Wolf, H., and Bornkamm, G.W (1980). Proc. Natl. Acad. Sci. U.S.A. 77:433-436.

6. Liu, C.-P., Slate, D.L., Gravel, R., and Ruddle, F.H. (1979). Proc. Natl. Acad. Sci. U.S.A. 76:4503-4506.

7. Sandri-Goldin, R.M., Goldin, A.L., Levine, M., and Glorioso, J.C. (1981). Mol. Cell. Biol. 1:743-752.

8. Schaffner, W. (1980). Proc. Natl. Acad. Sci. U.S.A. 77:2163-2167.

9. Klobutcher, L.A., and Ruddle, F.H. (1979). Nature 280:657-660.

10. Scangos, G.A., Huttner, K.M., Juricek, D.K., and Ruddle, F.H. (1981). Mol. Cell. Biol. 1:111-120.

11. Perucho, M., Hanahan, D., and Wigler, M. (1980). In Ninth Annual ICN-UCLA Symposia, Animal Virus Genetics. (eds.) Fields, B, and Jaenich, R. (Academic Press, New York), pp. 155-169.

12. Perucho, M., Hanahan, D., and Wigler, M. (1980). Cell 22:309-317.

13. Robbins, D.M., Ripley, S., Henderson, A., and Axel, R. (1981). Cell 23:29-39.
14. Smiley, J.R., Steege, D.A., Juricek, D.K., Summers, W.P., and Ruddle, F.H. (1978). Cell 15:455468.

15. Folger, K.R., Wong, E.A., Wahl, G., and Capecchi, M.R. (1982). Mol. Cell. Biol. 2:1372-1387.

16. de Saint Vincent, B.R., Delbruck, S., Eckhart, W., Meinkoth, J., Vitto, L., and Wahl, G. (1981). Cell 27:267-277.

17. Sandri-Goldin, R.M., Goldin, A.L., Holland, L.E., Glorioso, J., and Levine, M. (1983). Mol. Cell. Biol. 3:2028-2044.

18. Enquist, L.W., Vande Woude, G.F., Wagner, M., Smiley, J.R., and Summers, W.C. (1979). Gene 7:335-342.

19. Hanahan, D. (1983). J. Mol. Biol. 166:557-580.

20. Kit, S., Dubbs, D.R., Piekarski, L.J., and Hsu, T.C. (1963). Exp. Cell Res. 31:297-312.

21. Wigler, M., Silverstein, S., Lee, L.-S., Pellicer, A., Cheng, Y.-C., and Axel, R. (1977). Cell 11:223232.

22. Huttner, K.M., Barbosa, J.A., Scangos, G., Pravtcheva, D., and Ruddle, F.H. (1981). J. Cell Biol. 91:153-160.

23. de Saint Vincent, B.R., and Wahl, G.M. (1983). Proc. Natl. Acad. Sci. U.S.A. 80:2002-2006.

24. Small, J., and Scangos, G. (1983). Science 219:174-176.

25. Campione-Piccardo, J., and Rawls, W.E. (1981). Can. J. Microbiol. 27:1123-1128.

26. Pellicer, A., Wigler, M., Axel, R., and Silverstein, S. (1978). Cell 14:133-141.

27. Reyes, F.G., McLane, M.W., and Hayward, G.S. (1982). J. Gen. Virol. 60:209-224.

28. Roberts, J.M., and Axel, R. (1982). Cell 29:109119.

29. Southern, E.M. (1975). J. Mol. Biol. 98:503-517.

30. Goldin, A.L., Sandri-Goldin, R.M., Levine, M., and Glorioso, J.C. (1981). J. Virol. 38:50-58. 